Special Issue of the 8th International Advances in Applied Physics and Materials Science Congress (APMAS 2018)

\title{
A Multisource Power Management Interface for Energy Harvesting Circuits
}

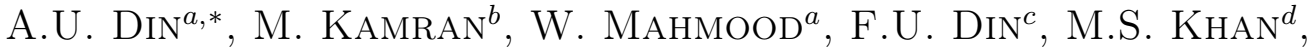 \\ M.T. GUL ${ }^{e}$ AND M.A. ABID ${ }^{f}$ \\ ${ }^{a}$ Material Synthesis \& Characterizations (MSC) Lab-Electronics Division, Department of Physics, \\ Fatima Jinnah Women University (FJWU), The Mall, Rawalpindi-Pakistan \\ ${ }^{b}$ Department of Electrical Engineering, COMSATS Wah Campus, Wah Cantt-Pakistan \\ ${ }^{c}$ Department of Physics, G.C University Faisalabad, Sahiwal Campus-Pakistan \\ ${ }^{d}$ Department of Electrical Engineering, Lahore Leads University, Lahore-Pakistan \\ ${ }^{e}$ Department of Electrical Engineering, Sharif University of Technology, Lahore-Pakistan \\ ${ }^{f}$ Institute of Computing and Information Technology, Gomal University, D.I. Khan-Pakistan
}

\begin{abstract}
In this paper, an efficient power management interface has been investigated using different green energy sources which are piezoelectric, radio frequency and thermal energy transducers. The piezoelectric transducer drives the rectifier. The radio frequency and thermal energy transducers drive Dickson charge pump and boost converter, and then a high-level voltage level selector circuit is used for the selection of the highest voltage level. Simulation results show that by using an efficient rectifier the efficiency of $80 \%$ can be achieved when the piezoelectric source is used. Moreover, the adaptive power management output the highest voltage level when any of the input sources is active. It is simulated using $180 \mathrm{~nm}$ CMOS structure and is implemented using discrete components.
\end{abstract}

DOI: 10.12693/APhysPolA.135.946

PACS/topics: energy harvesting, power management circuits, rectifiers

\section{Introduction}

For last few decades, the demand for self-powered electronics has been tremendously increased and the advances being made in wireless technology, and sensors are being developed that can be placed everywhere. However, because these sensors are wireless, they require their own power supply which in most cases is the battery, but the problem is its charging. The issue can be potentially alleviated through the use of power harvesting devices. By combining energy from the multiple sources, it is possible to increase the overall system and reliability. Also, there is a need to have a reconfigurable, universal energy harvesting system that can interface with any harvester [1-4].

S. Bandyopadhyay et al., envisioned a multi-input system using three different sources which are photovoltaic, thermal energy (TE) and piezoelectric (PE) and refine the voltage of transducers using conventional techniques like solar boost, thermal boost and PE buck-boost respectively and did not extract the maximum of energy from the transducers. The other drawback is its large size of $5 \times 5 \mathrm{~mm}^{2}$ [1]. In [2], a specific purpose aircraft health monitoring (AHM) system is proposed. It is based on two energy sources which are PE and TE using two external ultracapacitors (UC) for storage. Bulky UC acts as a battery. Highly flexible architecture with multiple sources is needed to deal with large variation in

*corresponding author; e-mail: ammad@fjwu.edu.pk environmental conditions. In this work, one such system is discussed that combines energy from radio frequency $(\mathrm{RF})$, thermoelectric (TE) and PE sources and provides the regulated voltage $1.8 \mathrm{~V}$ to power up the devices. The system has to supply for a variety of different energy sources. In this system, the power converters have to deal with a wide range of input voltages and if all the inputs are activated simultaneously, the highest voltage level will be selected by the high-level voltage level selector (HVLS) circuit.

\section{Design methodology}

The efficient architecture of multisource power management interface for energy harvesting circuits is shown in Fig. 1. The systems consist of the self-resonant rectifier (SRR), Dickson charge pump (DCP) [5], and a boost converter (BST) [6] sourced by the piezoelectric (PZ), $\mathrm{RF}$ and TE transducers respectively. These three rectified voltages are then fed into the high voltage level selector circuit which selects the highest voltage source and then a stabilizer is used to provide permanent $1.8 \mathrm{~V}$ output voltage.

\subsection{Proposed SRR rectifier circuit}

The block diagram of the proposed SRR rectifier is shown in Fig. 2a. It shows the voltage multiplier (VM), the pulse generator $(\mathrm{PG})$, symmetric flipping circuit, energy transfer (ET-En) enable circuit and a rectifier. The large quantity of available charge from the transducer is lost in charging and discharging the transducer capacitor. In this proposed design, an effort has been made to increase its power extraction capability close to $100 \%$. 


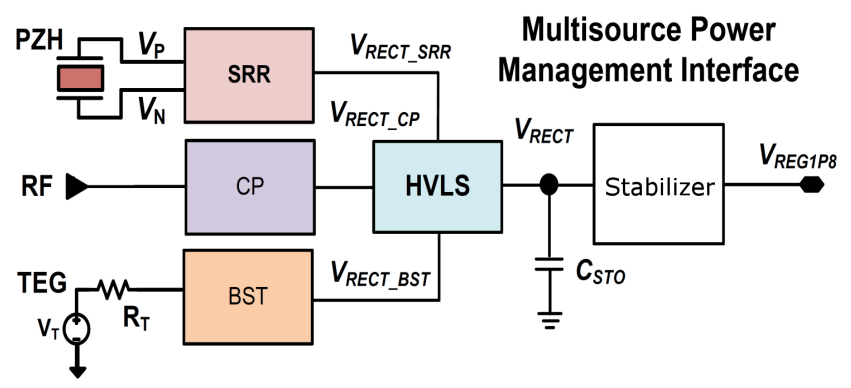

Fig. 1. Multisource source power management architecture.

(a)

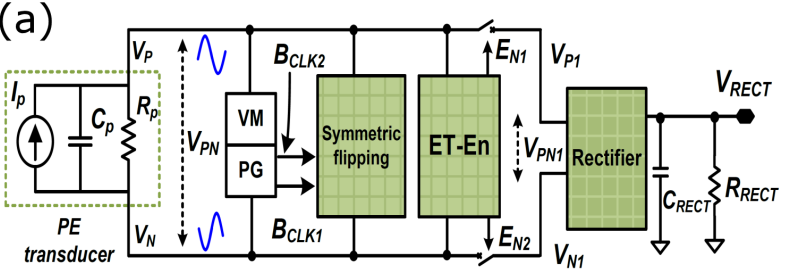

(b)

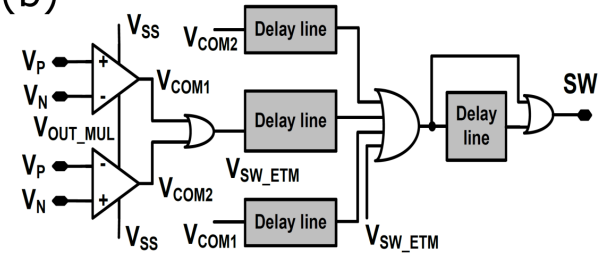

Fig. 2. (a) Block diagram of the proposed self-resonant rectifier (b) Proposed energy transfer enable (ET-En) technique.

The extraction of power relies on switching to discharge the internal capacitor of the transducer. Several techniques have been reported in the literature to improve the power extraction [4]. In this architecture symmetric flipping technique is adapted to extract the maximum power from the transducer. An energy transfer enables ET-En circuit shown in Fig. 2b is introduced. When the energy from the transducer is high enough the ET-En circuit turns on the $E_{N 1-2}$ switches to transfer the input energy at the output. The Dickson charge pump [5] and a boost converter circuit [6] is used for the $\mathrm{RF}$ and TE respectively.

\subsection{Proposed high voltage level selector circuits}

The schematic diagram of the high voltage level selector (HVLS) is shown in Fig. 3. A comparator using common gate topology detects the higher voltage level and then is fed into the gate of PMOS M1-M6 to select the highest voltage. The highest level is selected in two different stages. In stage 1 , the highest voltage level is selected between $V_{I N 1}$ and $V_{I N 2}$ and then between $V_{I N 2}$ and $V_{I N 3}$ called $V_{H 12}$ and $V_{H 23}$. After selecting the highest voltage levels of $V_{H 12}$ and $V_{H 23}$, then these voltage levels are fed into the next comparator to select the highest level between these two.

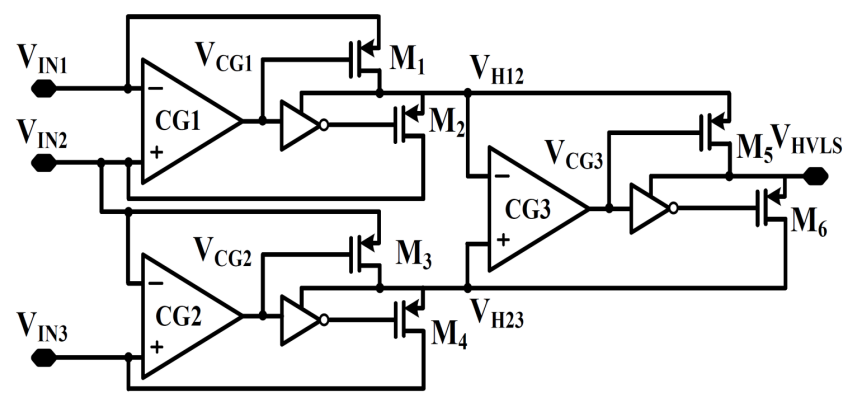

Fig. 3. Schematic diagram of the HVLS circuit.

\section{Results and discussion}

The proposed architecture is shown in Fig. 2 and is implemented using $180 \mathrm{~nm}$ CMOS structures. This rectifier is simulated with the transducer parameters such that $I_{p}=100 \mu \mathrm{A}, C_{p}=130 \mathrm{nF}, f_{p}=200 \mathrm{~Hz}$, and $R_{p}=1 \mathrm{M} \Omega$. The inductor coils $L_{1-2}$ in symmetric flipping circuit have the inductance of $1 \mathrm{mH}$. The simulation results of SRR rectifier are shown in Fig. 4a.
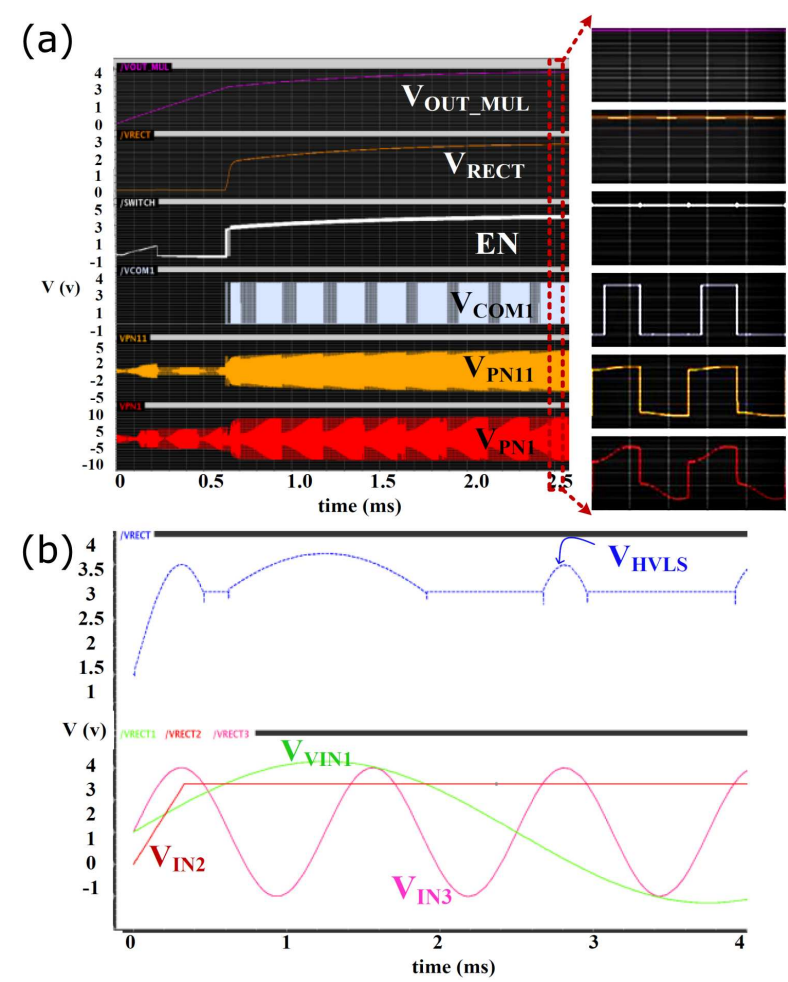

Fig. 4. (a) Simulation results of the SRR rectifier (b) Simulation results of the HVLS circuit.

It shows the input voltage $V_{P N}$, voltage after ET-En circuit $V_{P N 1}$, the output of $\mathrm{VM}$ and the output voltage $V_{R E C T}$. When clock pulses changes, $V_{P N}$ and $V_{P N 1}$ changes polarity and the measured flipping efficiency is $77 \%$. The output voltage $V_{R E C T}$ of the proposed rectifier is $3.71 \mathrm{~V}$ and the output power is $809 \mu \mathrm{W}$. The 
maximum extracted power for the proposed rectifier is $1017 \mu \mathrm{W}$, which reveals that their power conversion efficiency is $80 \%$. The simulation results of HVLS circuit are shown in Fig. 4b. There are three different levels of inputs and then the highest level is at the top which is $V_{H V L S}$.

\section{Conclusion}

In this study, three different sources which are piezoelectric, radio frequency and thermal energy are used and drives the SRR rectifier, Dickson CP and boost converter circuits respectively for conditioning and then a high voltage level selector circuit is proposed which selects the highest voltage level. A new type of rectifier is also proposed and simulated and found that the efficiency of $80 \%$ can be achieved using PE transducer is used by adopting an energy transfer enable circuit. It is simulated in PSPICE simulator using $180 \mathrm{~nm}$ CMOS structures. Simulated results show that the adaptive power management can be used when any of the input is active and $1.8 \mathrm{~V}$ is received permanently.

\section{Acknowledgments}

One of the authors (Amad Ud Din) would like to thank the Higher Education Commission (HEC) Pakistan for the travel grant funds.

\section{References}

[1] S. Bandyopadhyay, A.P. Chandrakasan, IEEE. J. Solid-State Circ. 47, 2199 (2012).

[2] C. Vankecke, L. Assouère, A. Wang, P. DurandEstèbe, F. Caignet, J. Dilhac, M. Bafleur, IEEE. Trans. Power Electron. 30, 3215 (2015).

[3] A. Klinefelter, N.E. Roberts, Y. Shakhsheer, P. Gonzalez, A. Shrivastava, A. Roy, K. Craig, M. Faisal, J. Boley, S. Oh, Y. Zhang, D. Akella, D.D. Wentzloff, B.H. Calhoun, in: Proceedings of IEEE International Solid-State Circuits Conference - (ISSCC) Digest of Technical Papers, San Francisco 2015, p. 384.

[4] Y.K. Ramadass, A.P Chandrakasan, IEEE. J. SolidState Circ. 45, 189 (2010).

[5] M. Muramatsu, H. Koizumi, in: Proceedings of IEEE International Conference on Sustainable Energy Technologies (ICSET), Kandy, Sri Lanka 2010, p. 1.

[6] Y.K. Ramadass, A.P. Chandrakasan, IEEE J. SolidState Circ. 46, 333 (2011). 